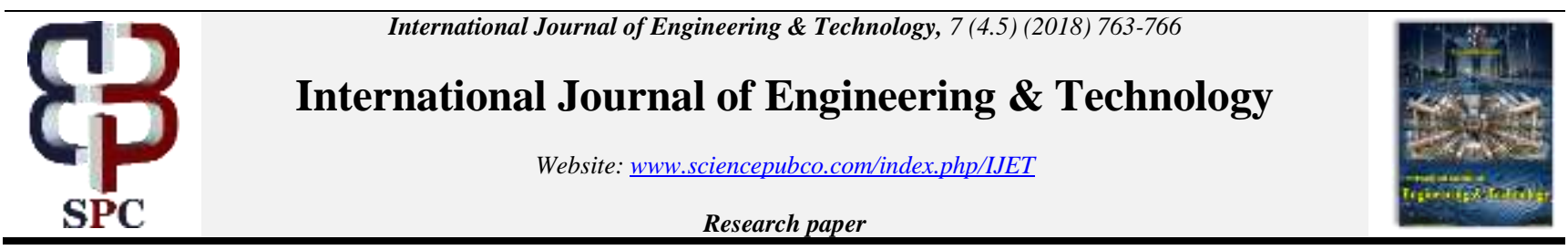

\title{
Designing controller for single area power plant using model order reduction method
}

\author{
Gauri Chandra ${ }^{1} *$, Lini Mathew ${ }^{2}$, Richa Negi ${ }^{3}$ \\ ${ }^{1}$ ME Scholar Electrical Engineering Department NITTTR, Chandigarh \\ ${ }^{2}$ Associate Professor Electrical Engineering Department NITTTR, Chandigarh \\ ${ }^{3}$ Associate Professor Electrical Engineering Department MNNIT Allahabad \\ *Corresponding author E-mail: chandragauri11@ gmail.com
}

\begin{abstract}
A two-step method for designing controller for a single area power plant (SAPP) is proposed. In the first step, a lower-order (lth-order) model for a higher-order (hth-order) power plant is obtained using dominant pole retention and Padé approximation method and a controller is designed for this model. This controller is then implemented to original higher-order power system in the next step. The method preserves stability and disturbance rejection is also satisfactory.
\end{abstract}

Keywords: Use about five key words or phrases in alphabetical order, Separated by Semicolon.

\section{Introduction}

Mathematical modelling of large engineering systems often leads to higher order differential equations. The analysis of such high order system is not only difficult but costly for online implementation. Moreover, designing a controller of original model seems to be difficult or even impossible. Most of the control systems designs are based on second-order approximation to whatever order the process happen to be, and these control system work, usually quite well. It is, therefore desirable and sometimes necessary to replace these higher-order systems (HOS) by a lower-order model (LOM). A large variety of methods have been proposed both in frequency as well as in time domain [1], [2].

A very powerful method involving simple algebraic calculation is Padé approximation [3] method. This method, however, has the drawback that the model derived using this method sometimes produces unstable models for stable HOS. Many methods have appeared in literature such as Routh approximation and impulse energy approximation [4], [5] to ensure stability.

Another method for deriving LOM is Krylov subspace method [6]. It can handle systems of several thousand orders but it has a drawback that it does not ensure stability of LOM. Recently, $H_{\infty}$ and fuzzy based methods have also been presented [7]. An important group of reduction method is dominant pole retention and pole clustering $[8,9]$. These methods ensure stability of LOM and are simple to implement. Methods based on ISE minimization have also appeared in the literature [10]. In these methods, Pareseval's theorem is used which changes ISE in denominator and numerator of lower-order transfer function.

In this paper, a mixed method is proposed. In the first step, a stable LOM, using dominant pole retention and Padé approximation is determined. The controller is derived corresponding to this LOM and then implemented to HOS in the second step. The denominator parameters of LOM are obtained using dominant pole retention method and numerator parameters of LOM are deter- mined by Padé approximation. The method is applied for designing a controller for a single area power plant.

\section{Problem formulation}

Suppose the HOS is given by $G_{h}(s)$. In the first step, a lowerorder controller $C_{l}(s)$ is derived for this $G_{h}(s)$ represented by:

$$
G_{h}(s)=\frac{d_{1} s^{h-1}+d_{2} s^{h-2} \ldots+d_{h}}{s^{h}+c_{1} s^{h-1}+c_{2} s^{h-2} \ldots+c_{h}}
$$

Or

$$
G_{h}(s)=\frac{d_{1} s^{h-1}+d_{2} s^{h-2} \ldots+d_{h}}{\left(s+p_{h 1}\right)\left(s+p_{h 2}\right) \ldots\left(s+p_{h n}\right)}
$$

Where $-p_{h 1},-p_{h 2} \ldots,-p_{h n}$ are poles of HOS and a LOM of the following form is to be derived.

$$
G_{l}(s)=\frac{\hat{d}_{1} s^{l-1}+\hat{d}_{2} s^{l-2} \ldots+\hat{d}_{l}}{\left(\mathrm{~s}+p_{d 1}\right)\left(\mathrm{s}+p_{d 2}\right) . .\left(s+p_{d l}\right)}
$$

Or

$$
G_{l}(s)=\frac{\hat{d}_{1} s^{l-1}+\hat{d}_{2} s^{l-2} \ldots+\hat{d}_{l}}{s^{r}+\hat{c}_{1} s-\hat{c}_{2} s^{l-2} \ldots+\hat{c}_{l}}
$$


Where $-p_{d 1}, \ldots,-p_{d l}$ are the dominant poles of the LOM.

\subsection{Determination of denominator of LOM}

The dominant poles are those poles which are closer to $j \omega$ axis. The denominator $D_{l}(s)$ of the ROM is determined by retaining $\mathrm{r}$ dominant poles of (2) in the form as shown in (3). Once the dominant poles $\left(\mathrm{s}+p_{d 1}\right)\left(\mathrm{s}+p_{d 2}\right) . .\left(s+p_{d l}\right)$ are determined, the denominator $D_{l}(s)$ is easily obtained as:

$$
D_{l}(s)=s^{l}+\hat{c_{1}} s^{l-1}+\hat{c_{2}} s^{l-2}+\ldots+\hat{c_{l}}
$$

\subsection{Determination of numerator of LOM}

Padé approximation is used to determine numerator of LOM $G_{h}(s)$ as given in (1) is expanded in Taylor series as:

$$
\begin{aligned}
& G_{h}(s)=t_{1}+t_{2} s+\ldots+t_{h} s^{h-1}+\ldots \quad \quad(\text { about } s=0) \\
& \quad=M_{1} s^{-1}+M_{2} s^{-2}+\ldots+M_{h} s^{-h}+\ldots \quad(\text { about } s=\infty)
\end{aligned}
$$

Where $t_{1}, t_{2}, t_{3}, \ldots$ and $\hat{M}_{1}, \hat{M}_{2}, \hat{M}_{3} \ldots$ are the time moments (TM) and Markov parameters (MP) of the HOS. Similarly $G_{(}(s)$ is expanded in Taylor series as:

$$
\begin{aligned}
G_{t}(s) & \left.=\hat{t}_{1}+\hat{t}_{t} s+\ldots+\hat{t}_{s} s^{\prime-1}+\ldots \quad \text { (about } s=0\right) \\
& \left.=\hat{M}_{1} s^{-1}+\hat{M}_{2} s^{-2}+\ldots+\hat{M}_{1} s^{-1}+\ldots \quad \text { (about } s=\infty\right)
\end{aligned}
$$

Where $\hat{t}_{1}, \hat{t}_{2}, \hat{t}_{3}, \ldots$ and $\hat{M}_{1}, \hat{M}_{2}, \hat{M}_{3} \ldots$ are TM and MP of the lowerorder model. The problem is formulated for 1 even. For 1 odd, it may be formulated in the same way.

For the LOM to be stable, the 1 equations as given in (8) are to be satisfied.

$$
\hat{t}_{j}-t_{j}=0 \text { and } \hat{M}_{j}-M_{j}=0 \quad(j=1,2, \ldots, l / 2)
$$

It may be verified that the time moments and the Markov parameters are related with the numerator and denominator coefficients as

$$
\hat{d}_{l+1-j}=\sum_{k=1}^{j} t_{k} \hat{c}_{l-j+k} \text { and } \hat{d}_{j}=\sum_{k=1}^{j} M_{k} \hat{c}_{j-k} \text { for } j=1,2,3, \ldots, l / 2
$$

Now, matching required number of TM and MP in accordance with (8) and (9) of the HOS and LOM, numerator coefficients $\hat{d}_{1}, \hat{d} 2, \ldots \hat{d}_{l}$ LOM is computed as:

$$
N_{l}(s)=s^{l}+\hat{d} s^{l-1}+\hat{d} s^{l-2}+\hat{d}_{s} s^{l-3}+\ldots . .+\hat{d}_{l}
$$

\subsection{Controller design}

Consider a unity feedback control system [11] as shown in Fig. 2.1. In the classical approach, the controller $C(s)$ is designed by specifying the desired closed-loop (CL) transfer function $T_{d}(s)$ and equating it to the CL transfer function of the system. Then, this equation is solved for finding the controller.

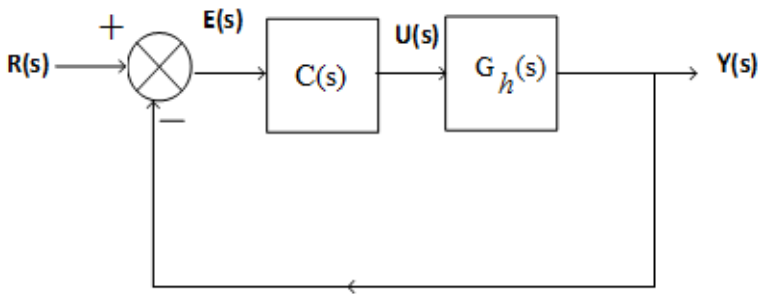

Fig. 2.1: Controller Configuration.

The transfer function of the CL system for Fig. 2.1 is obtained as $T_{o}(s)=\frac{C(s) G_{h}(s)}{1+C(s) G_{h}(s)}$

Since overall all transfer function is chosen as desired model

$T_{d}(s)=\frac{C(s) G_{h}(s)}{1+C(s) G_{h}(s)}$

Solving for controller ${ }^{c(s)}$

$C(s)=\frac{T_{d}(s)}{G_{h}(s)\left[1-T_{d}(s)\right]}$

If, ${ }^{G_{h}(s)}$ is reduced to ${ }^{G_{l}(s)}$ by a suitable reduction method, as shown in Fig. 2.2, for the same desired transfer function, the lower-order controller configuration ${ }^{C_{l}(s)}$ is expressed as

$C_{l}(s)=\frac{T_{d}(s)}{G_{l}(s)\left[1-T_{d}(s)\right]}$

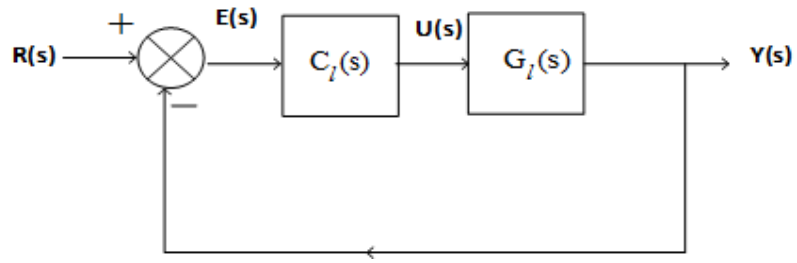

Fig. 2.2: CL Control with $G_{l}(s)$ and $C_{l}(s)$.

The overall CL transfer function combining LOM and lower-order controller is expressed as

$T_{o l}(s)=\frac{C_{l}(s) G_{l}(s)}{1+C_{l}(s) G_{l}(s)}$

The CL control configuration combining HOS and lower-order controller is shown in Fig.2.3

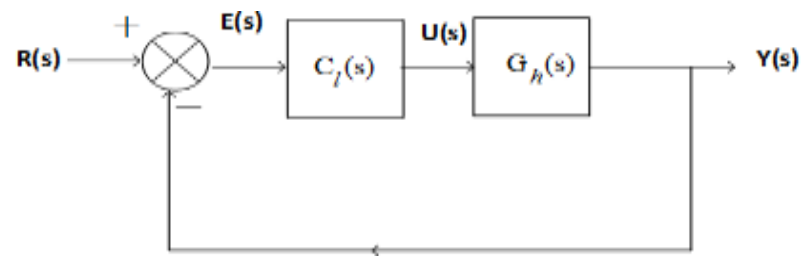

Fig. 2.3: CL Control with $G_{h}(s)$ And $C_{l}(s)$

The overall transfer function of Fig. 2.3 is

$$
T_{o h}(s)=\frac{C_{l}(s) G_{h}(s)}{1+C_{l}(s) G_{h}(s)}
$$


Following numerical example is used to describe the step-by-step procedure of the proposed algorithm.

\section{Numerical example}

Consider a linearized SAPP [12] as shown in Fig. 3.1 In the Fig. 3.1, the governor dynamics is given by ${ }_{g o v}(s)=1 /\left(T_{g o v} s+1\right)$, load and machines dynamics is given by ${ }_{l m}(s)=K_{p} /\left(T_{l m} s+1\right)$, and the droop characteristics is given by $1 / R$. The non-reheated turbine dynamics is represented as $G_{m t}(s)=1 /\left(T_{n t} s+1\right)$ with ${ }^{\Delta P_{d i t}}$ (in p.u. MW) represented as the load disturbance. After appropriate reduction, Fig. 3.1 takes the form as shown in Fig. 3.2.

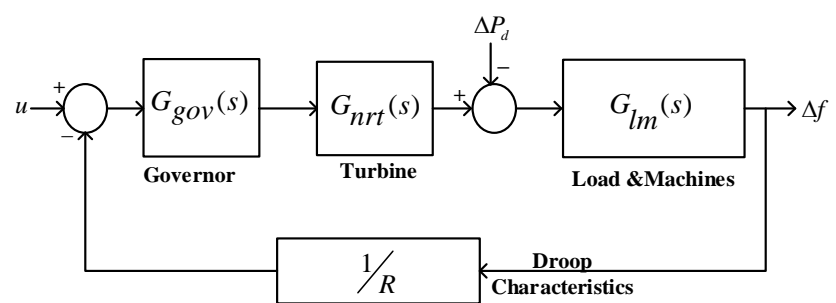

Fig. 3.1: Linearized SAPP.

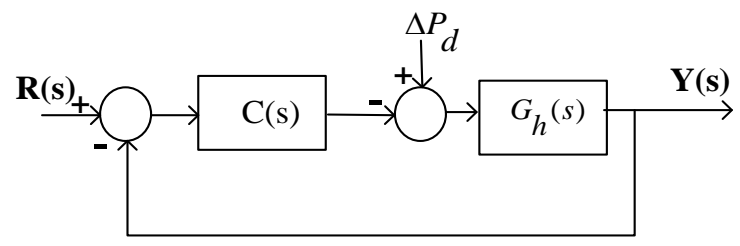

Fig. 3.2: Controller Configuration of SAPP.

With ${ }^{K_{p}}=120, T_{l m}=20, T_{m i t}=0.3, T_{g o v}=0.08,{ }^{R}=2.4$, the transfer function $G_{h}(s)$ turns out to be

$$
\begin{aligned}
& G_{3}(s)=\frac{250}{s^{3}+15.88 s^{2}+42.46 s+106.2} \\
& =\frac{250}{(s+13.2858)(s+1.2971+j 2.5122)(s+1.2971-j 2.5122)}
\end{aligned}
$$

In the first step, a LOM for $1=2$ is obtained retaining two dominant poles ${ }^{(s+1.2971 \pm j 2.5122)}$ of (17), the ${ }^{D_{I}(s)}$ as given in (5) becomes

$$
D_{l}(s)=s^{2}+2.5948 s+7.994
$$

The numerator polynomial of LOM is obtained using (9) and (10) as

$$
N_{l}(s)=18.82
$$

Thus, the LOM turns out as

$$
G_{2}(s)=\frac{18.82}{s^{2}+2.5948 s+7.994}
$$

In the second step, ${ }^{c_{(s)}(s)}$ is derived by choosing ${ }^{T_{d}(s)}$ in such a way that it satisfies the frequency domain control specifications (damping ratio $\left({ }^{5}\right)$, undamped natural frequency $\left({ }^{\omega}{ }^{\omega}\right)$, etc.). Currently, a second-order ${ }^{T(s)}$ is chosen having $\zeta=0.7$ and natural frequency

\footnotetext{
${ }^{\omega}=1.5 \mathrm{rad} / \mathrm{sec}$. Thus
}

$T_{d}(s)=\frac{2.25}{s^{2}+2.1 s+2.25}$

The ${ }^{C_{l}(s)}$ is derived using (14) as

$$
\mathrm{C}_{2}(s)=\frac{0.1196\left(s^{2}+2.5947 s+7.994\right)}{s^{2}+2.1 s}
$$

Now, ${ }^{T_{a}}$ and ${ }^{T_{\omega}}$ are obtained using (15) and (16) respectively as

$T_{o l}=\frac{2.2508}{s^{2}+2.1 s+2.2508}$

$$
T_{\text {oh }}=\frac{29.9}{s^{3}+15.38 s^{2}+27.9 s+29.9}
$$

The Fig.3.3 contains the step responses of (17) and (20). It is seen that they quite close. The Fig. 3.3 also includes responses of $T_{o l}$ and ${ }^{T_{\omega}}$ also. It is clearly found that they are also close to each other.

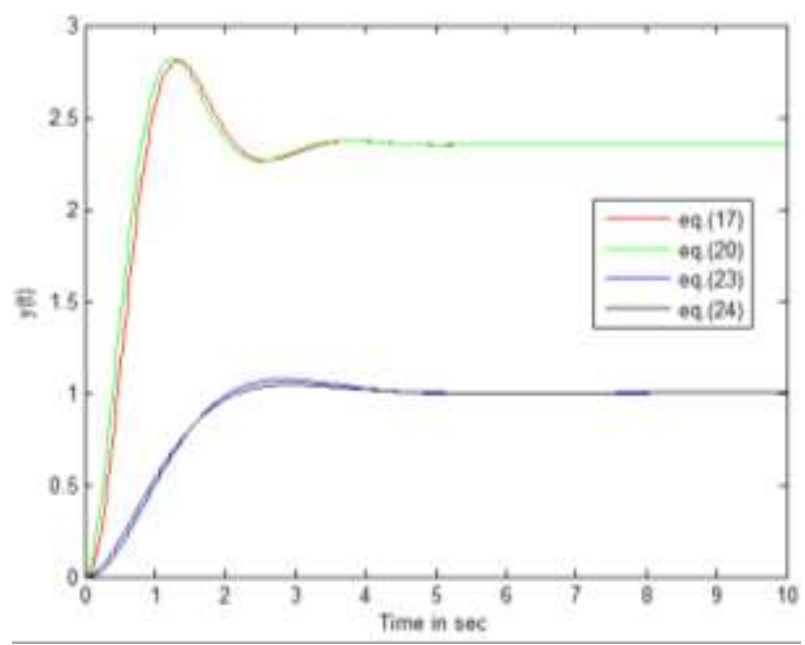

Fig. 3.3: Step Responses Of HOS and LOM and ${ }^{T_{o r}} T_{\star}$.

A disturbance of ${ }^{\Delta P_{d u}}=-0.01$ is applied at $\mathrm{t}=2$ sec. as shown in Fig. 3.2. The response with disturbance and without disturbance is plotted in Fig. 3.4. It is clearly observed that the disturbance rejection is also satisfactory.

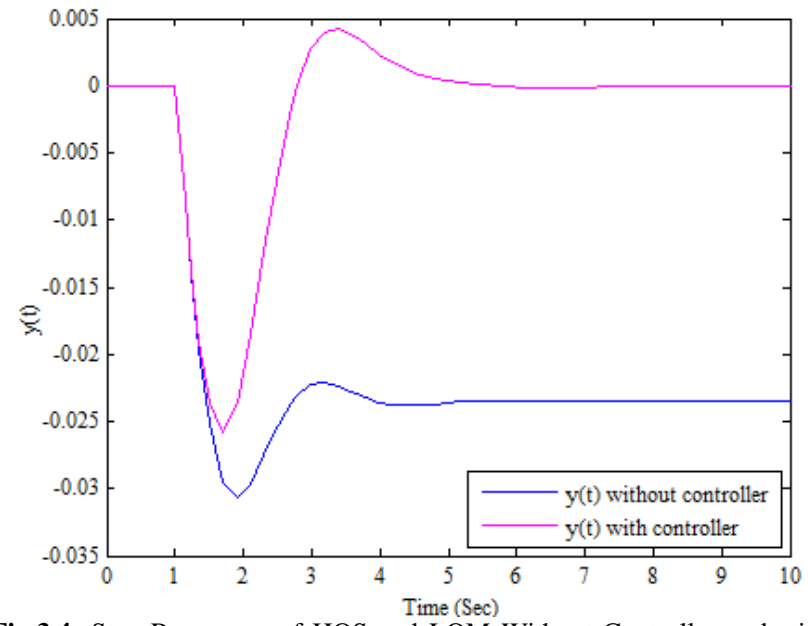

Fig.3.4: Step Responses of HOS and LOM Without Controller and with Controller. 


\section{Conclusion}

This note presents a two step procedure to design a controller for a single area power plant using concept of model reduction. In the first step, the HOS is simplified to a LOM. The dominant poles are retained for the purpose of deriving denominator of LOM while numerator of LOM is derived with help of Padé approximation. In the second step, the controller is designed for the LOM obtained in step 1 and then impelented to HOS in step two. As seen by the step resonses, the controller design shows satisfactory performance. A disturbance of $-0.01 \mathrm{p}$. u. $\mathrm{Mw}$ is injected to the system to check the performance of proposed controller 'with and without controller' situations.. The disturbance rejection is also found satisfactory.Methods based on intelligent conrollers have also been suggested in literature [14]. It would be interesting to compare the performance of proposed controller with other intelligent controllers and the problem is open for investigation.

\section{References}

[1] B. Bandyopadhyay and S.S. Lamba, "Time-domain Pade approximation and modal-Padé method for multivariable Systems", IEEE Trans. Circuits Syst., Vol. CAS-34, No. 1, 1987.

[2] Chiu T-Y, "Model reduction by the low frequency approximation balancing method for unstable systems" IEEE Trans. on Auto. Contr. vol. 41(7), pp.995-997, 1996.

[3] Chuang C., "Homographic transformation for the simplification of discrete-time functions by Padé approximation", Int. J. Contr., Vo. 22, pp. 721-729, 1975.

[4] MF Hutton and B Friedland, "Routh approximation for Reducing Order ofLlinear, Time-Invariance System” IEEE Trans. On Auto. Contr. vol. 20, pp.329-337, 1975.

[5] TN Lucas "Scaled impulse energy approximation for model reduction" IEEE Trans. on Auto. Contr. vol. 33(8), pp.791-793, 1988

[6] B Salimbahrami and B Lohmann, "Krylob-subspace methods in linear model order reduction: Introduction and invariance properties." Institute of automation, university of Bremen, Germany.

[7] X Su, “ $H_{\infty}$ - model order reduction of takagi-sugeno fuzzy stochastic sytems" IEEE Trans. Syst. Man, and Cyber vol(42): pp 1574 $1585,2012$.

[8] CB Vishwakarma and R Prasad, "Clustering method for reduction order of liner system using Pade Approximation" IETE Journal of Research vol. 54: pp. 326-330, 2008.

[9] A Narain, D Chandra and R K Singh, "Model order reduction using fuzzy C-means clusitering" Transactions of the Institute of Measurement and Control, pp-1-7, 2014.

[10] V Singh, D Chandra and H Kar, "Optimal Routh Approximants through Integral Squared Error Minimization: Computer-Aided Approach", IEEE Proc. Contr. Theory Appl. Vol. 151, pp-53-58, 2004.

[11] L.A. Aguirre, "Designing Controller by means of model reduction technique", Electronic letter, vol-3, 1993.

[12] S. Saxena, YV Hote. "Load frequency control in power systems via internal model control scheme and model-order reduction." IEEE T POW Sys; 28: 2749-2757. 2013.

[13] B. Sonkar, D. Kumar and P. Samuel, “ A Modified two degree of freedom-integral model control configuration for load frequency control of a single area power system”, Turk. J. Elec. Eng. \& Comp Sci, Vol.-25: pp 4624-4635, 2017.

[14] A. Demiroren, N. S. Senegor, and N. S. Zeynelgil, “Automatic generation control by using ANN techniques" Electro. POW. Compo Sys.,vol. 29, pp. 883-896, 2001. 